\title{
COMMENTS ON THE MATHFIT PROGRAMME
}

\author{
Anthony Karel Seda
}

\section{Introduction}

Readers of the Bulletin who are not members of the London Mathematical Society (LMS), and even some LMS members, may be unaware of the UK's Mathfit programme, see [1, 2, 3, 4]. Mathfit is short for "Mathematics for Information Technology" and is the result of collaboration between the LMS and the EPSRC (Engineering and Physical Sciences Research Council). In fact, Mathfit is a programme of study and research of three years duration which started in the summer of 1996. Its function is to promote interaction between mathematicians and those involved in computing and information technology, to the mutual benefit of both communities. Indeed, its objectives as stated in [4] are: "(i) To develop a climate in which new links between mathematics and information science can flourish so as to stimulate and support high quality research in both fields. (ii) To provide the training needed to enable high-quality research students to move into these new areas and to ensure that the wider community is aware of recent developments and are trained in the contemporary scientific and mathematical techniques to understand and implement them. (iii) To enable senior scientists of high quality in mathematics and computer science to work in collaboration and to develop research programmes in new areas complementary to their own." Mathfit is, therefore, broadly modelled on the Logfit (Logic for Information Technology) initiative mounted by the UK's former Science and Engineering Research Council. Logfit was successful in encouraging logicians to become involved in IT, and Mathfit, it appears, is attempting to widen the input on the theoretical side to include most areas of mathematics, not just logic. 
The mechanisms by which Mathfit sets out to achieve its aims, quoting [4], are: “(1) The provision of Research Proposals and Visiting Fellowships (particularly those which seek to transfer personnel between complementary mathematics departments and computer science departments or vice versa). (2) Workshops and Summer Schools which will address the Mathfit aims, particularly in those areas which are identified as priorities" (see Section 3).

My objectives in writing this short note are to bring the Mathfit programme to the notice of readers, especially those who have an interest in the interaction between mathematics and computer science, and also to highlight those areas of mathematics which are deemed to be important by those responsible for Mathfit. It seems to me that Mathfit is a major programme and is significant in identifying important areas of research. It follows, therefore, that it should also be of interest to those involved in curriculum development in mathematics in relation to IT. Of late, this issue has become important due to the rise in prominence of IT in this country. Perhaps the lead and framework provided by those behind Mathfit can be used to advantage in this country in promoting the rôle of mathematics in the training of IT professionals. Indeed, an initiative of this sort here, on an appropriate scale, could be of considerable value in promoting the interaction between Mathematics and IT in general.

\section{Background}

An LMS survey in 1995 of heads of departments of computer science, see [2], set out to identify the requirement for a series of instructional conferences and to solicit suggested topics. The responses included "abstract interpretation, adaptive algorithms, asymptotic analysis, automated deduction and proof theory, category theory, combinatorial optimisation, computational complexity, computational geometry, constructive mathematics, data security, domain theory, genetic algorithms, graph theory, information theory, kinematics for students working in robotics, lambda calculus, logic, machine learning, mathematical analysis of computer vision, mathematical analysis of search techniques, mathematics of concurrency, mathematics of constraints and optimisation, model 
theory, model theory and specification, neural networks, number theory for information security, numerical analysis, parallelism and concurrency, proof theory, proofs and types, relative complexity of proof systems, signal processing, structural complexity, symbolic computation, type theory for computer algebra, types, and interactive proofs".

Similarly, a survey of departments of mathematics and computing set out to "solicit proposals for instructional workshops for researchers, particularly postgraduate students, in order to provide instruction in topics from mathematics and logic which are of importance in computer science. This elicited firm proposals from 15 groups in the following areas: algebraic aspects of circuit design, algebraic foundations of algorithms, Boolean function complexity, category and type theory, computer algebra and symbolic computation, computer vision, descriptive complexity theory, foundations and applications of pi-calculus, graph theory, mathematical analysis of networked and distributed systems, mathematical modelling of software systems, mathematics of machine learning, regular and systolic algorithm design, specification and implementation of concurrent systems, term graph rewriting". Details of the workshops which have taken place can be found in [3].

\section{Priority Areas Identified by Mathfit}

Based on the surveys described in the previous section certain areas were identified in [1, 4] as priorities for future work, as listed below. It was noted in [4] that "this list will evolve and changes to the list may be made in future years":

- Algorithms and Structures: combinatorics, complexity theory, discrete mathematics.

- Artificial intelligence, including learning, neural computation, planning and reasoning: differential geometry and non-linear dynamics, logics, probabilistic and statistical techniques.

- Complex, communicating and concurrent systems: dynamical systems, ergodic theory, functional analysis, process calculi and logics, stochastic systems.

- Computer graphics, robotics and vision: algebraic, computational and differential geometry, kinematics, topology. 
- Principles of programming languages, including semantics and language design: category theory, game theory, logic, ordered structures and domain theory, universal algebra.

- Networks, telecommunications and information security: cryptology, data encoding, digitisation, graph theory, information theory, number theory, probabilistic and statistical techniques, signal processing.

Space limitations do not permit anymore than the presentation of the bare facts, other than to emphasise the importance to educators, already noted, of this sort of information. However, in closing, it is worth noting that a remarkably wide range of mathematical tools and ideas is identified in the topics listed above. Maybe at one point in time "discrete mathematics" was sufficient background training for those involved in IT. One thing that is clearly apparent from the Mathfit programme is that that time has passed.

\title{
References
}

See http://www.epsrc.ac.uk:80/progs/technology/itcs/mfitcall.htm for all the following references.

[1] Mathfit.html

[2] Mathfit2.html

[3] Meetings.html

[4] Mfitcall.html

\author{
A.K. Seda \\ Department of Mathematics, \\ University College, \\ Cork, \\ Ireland.
}

email:aks@bureau.ucc.ie 